\title{
Activation of human $T$ cells by a tumor vaccine infected with recombinant Newcastle disease virus producing IL-2
}

\author{
MARKUS JANKE ${ }^{1,4}$, BEN PEETERS $^{2}$, HENG ZHAO $^{2}$, OLAV DE LEEUW $^{3}$, ROB MOORMAN $^{3}$, \\ ANNETTE ARNOLD $^{1}$, YVONNE ZIOUTA ${ }^{1}$, PHILIPPE FOURNIER ${ }^{1}$ and VOLKER SCHIRRMACHER ${ }^{1}$
}

${ }^{1}$ Division of Cellular Immunology, German Cancer Research Center, Im Neuenheimer Feld 280, D-69120 Heidelberg, Germany;

${ }^{2}$ Division of Virology, Central Institute for Animal Disease Control Lelystad (CIDC-Lelystad), P.O. Box 2004, 8203 AA Lelystad;

${ }^{3}$ Division of Infectious Diseases, Animal Sciences Group, Wageningen University and Research Centre, P.O. Box 65, 8200 AB Lelystad, The Netherlands; ${ }^{4}$ Division of Clinical Chemistry and Pharmacology, University Hospital, University of Bonn, Sigmund-Freud-Str. 25, D-53105 Bonn, Germany

Received May 2, 2008; Accepted June 19, 2008

DOI: 10.3892/ijo_00000070

\begin{abstract}
A new recombinant (rec) Newcastle disease virus (NDV) with incorporated human interleukin 2 (IL-2) as foreign therapeutic gene [rec(IL-2)] will be described. The foreign gene in rec(IL-2) did not affect the main features of NDV replication nor its tumor selectivity. Biologically active IL-2 was produced in high amounts by tumor cells infected with rec(IL-2). Tumor vaccine cells infected by rec(IL-2) stimulated human $\mathrm{T}$ cells to exert anti-tumor activity in vitro in a tumor neutralization assay. These effects were significantly increased when compared to vaccine infected by rec(-) virus without IL-2 gene. After incubation with rec(IL-2) infected tumor cells, T cells showed increased expression of the activation marker CD69 and produced increased amounts of IFN $\gamma$ when compared to T cells co-incubated with rec(-) infected tumor cells. CD8 T cells incubated with rec(IL-2) infected tumor cells showed upregulation of perforin, cell surface exposure of the degranulation marker CD107a and increased anti-tumor cytotoxic activity. Purified T cells from lymph nodes of head and neck squamous cell carcinoma (HNSCC) patients could be stimulated to secrete IFN $\gamma$ in an ELISPOT assay upon $40 \mathrm{~h}$ of stimulation with rec(IL-2) infected autologous tumor cells [ATV-rec(IL-2)] but not upon stimulation with rec(IL-2) infected allogeneic U937 tumor cells. This suggests direct activation of patient derived tumor antigen-specific memory T cells by ATV-rec(IL-2). In conclusion, the already inherent immunostimulatory properties of NDV could be further augmented by the introduction of the therapeutic gene IL-2. Active specific immunization of
\end{abstract}

Correspondence to: Professor V. Schirrmacher, Division of Cellular Immunology, D010, German Cancer Research Center (DKFZ), Im Neuenheimer Feld 280, D-69120 Heidelberg, Germany E-mail: v.schirrmacher@dkfz-heidelberg.de

Key words: interleukin 2, Newcastle disease virus, cancer vaccination, $\mathrm{T}$ cells, tumor therapy patients with ATV-rec(IL-2) should provide the microenvironment at the vaccination site with IL-2 and avoid side effects as seen after systemic IL-2 application.

\section{Introduction}

In recent years, reverse genetics technology enabled to generate recombinant strains from non-segmented negative strand RNA viruses such as Newcastle disease virus (NDV) (1). We previously established an NDV based autologous tumor vaccine (ATV-NDV), which consists of patient derived irradiated autologous tumor cells infected with the non-lytic NDV strain Ulster. Postoperative application of this vaccine caused prolongation of survival in several metastasizing animal tumor models (2). Improvements of survival (3) were also noted in clinical studies of colorectal carcinoma (4), breast cancer (5) and head and neck squamous cell carcinoma (HNSCC) (6). Clinical benefits were also noted in a recent study in glioblastoma multiforme (GBM) including one complete remission and prolongation of median overall survival (100 weeks versus 49 weeks in controls) (7).

The aim of this study was to introduce a foreign therapeutic gene into NDV to be able to generate a tumor vaccine, which upon infection with such virus would produce the therapeutic gene product locally. As a proof of concept, we will demonstrate activation of human $\mathrm{T}$ cells by a tumor vaccine infected with recombinant NDV producing human IL-2 [rec(IL-2)]. By infecting tumor cells with rec(IL-2) the IL-2 gene is amplified along with virus replication and its therapeutic gene product is released. While systemic application of IL-2 causes severe side effects associated with the vasculary leak syndrome, tumor targeted IL-2 was shown to be capable of eradicating established pulmonary and hepatic metastases in syngeneic mouse tumor models of melanoma (8) and colorectal carcinoma (9). It is likely that these effects were associated with $\mathrm{T}$ cell mediated anti-tumor immunity and that similar effects may be achieved with an IL-2 producing tumor vaccine. The advantages of using NDV as vector for gene transfer are its tumor-selectivity of replication (10) and its high safety profile $(11,12)$. This report will demonstrate a 
significant augmentation of anti-tumor cytotoxic activity upon stimulation of human $\mathrm{T}$ cells with a tumor vaccine infected with IL-2 producing NDV.

\section{Materials and methods}

Antibodies and chemicals. For FACS analysis the following monoclonal antibodies (mAbs) against human antigens were used at a dilution of 1:10: CD3-FITC/PerCP-Cy5.5, CD4Pacific blue, CD8-PerCP-Cy5.5/APC/FITC, CD107-PE, CD69-FITC/APC, Perforin-PE (Pharmingen, Hamburg, Germany). Mouse anti-HN monoclonal antibody (HN.B $\mathrm{mAb}, \mathrm{IgG} 2 \mathrm{a}$ ) and mouse anti-F (lcii mAb, IgG1) were kindly provided by Dr Ronald M. Iorio (Department of Molecular Genetics and Microbiology, University of Massachusetts, Medical School, MA). These antibodies were used at $1 \mu \mathrm{g} /$ $5 \times 10^{5}$ cells to detect NDV viral antigens or bispecific antibody binding on host cell surfaces. To detect the unconjugated anti-viral antibodies the cells were incubated with secondary mAb goat $\mathrm{F}(\mathrm{ab})_{2}$ anti-mouse Ig-RPE (1:100) obtained from Southern Biotechnology Associates, Inc. (Birmingham, AL). Intracellular staining with mouse antihuman $\mathrm{mAb}$ against perforin was performed after permeabilization and fixation of purified cells with a Cytofix/ Cytoperm Kit (BD Biosciences, Heidelberg, Germany) according to the manufacturer's protocol. Isotype controls were performed as suggested by the mAb suppliers. Bispecific antibody for simultaneous CD28 and viral HN binding (bsHN-CD28) was established and produced as described (13). Binding to cell surfaces of $0.6 \mu \mathrm{g} / \mathrm{ml}$ of bsHN-CD28 was detected by using $1 \mu \mathrm{g} / \mathrm{ml}$ mouse anti-flag polyclonal antibody (Sigma, Deisenhofen, Germany). ELISA experiments for the detection of human IFN- $\gamma$ and IL-2 (Pharmingen, Hamburg, Germany) were performed in accordance to the manufacturer's guidelines. MTS Cell Titer $96^{\circledR}$ Aqueous solution was purchased from Promega, Mannheim, Germany.

Cell lines and culture. All tumor cell lines were obtained from the Tumorbank DKFZ, Heidelberg, Germany. The human breast carcinoma cell line MCF-7 was maintained in DMEM containing 10\% inactivated FCS, 2 mM L-glutamine, $10 \mathrm{mM}$ HEPES, $100 \mathrm{U} / \mathrm{ml}$ penicillin and $100 \mu \mathrm{g} / \mathrm{ml}$ streptomycin. The human promonocytic lymphoma cell line U937 was cultured in RPMI-1640 containing 10\% inactivated FCS, $2 \mathrm{mM}$ L-glutamine, $10 \mathrm{mM}$ HEPES, $100 \mathrm{U} / \mathrm{ml}$ penicillin and $100 \mu \mathrm{g} / \mathrm{ml}$ streptomycin.

All reagents for cell culture were purchased from Gibco Life Technologies (Karlsruhe, Germany) except FCS, which was from Biochrom (Krefeld, Germany). Recombinant human IL-2 and IL-4 was obtained from PromoCell (Heidelberg, Germany). All cell lines were incubated at $37^{\circ} \mathrm{C}$ in a humidified atmosphere of $5 \% \mathrm{CO}_{2}$.

Autologous tumor cell culture. Tumor cell cultures from different HNSCC tumor patients were established as described previously (6) and kindly provided by Dr Christel Herold-Mende. In brief, tumor samples were mechanically dissected within $2 \mathrm{~h}$ after resection. The cell suspension was cultured in DMEM (Invitrogen, Karlsruhe, Germany) supplemented with $10 \%$ fetal calf serum (Biochrom, Berlin, Germany) and antibiotics. Only pre-tested serum batches from countries in which bovine spongiophorm encephalopathy has never been diagnosed were used. Mycoplasma contamination was excluded by 4',6-diamidino-2-phenylindole staining (Roche Diagnostics, Mannheim, Germany) and cells were routinely tested for lack of fungal and yeast contaminations. We characterized all short-term cultures for their epithelial origin by the immunohistochemical detection of tissue-specific markers, using antibodies recognizing a broad spectrum of cytokeratins, and for expression of MHC I molecules. Cells were stored in liquid nitrogen until use.

Plasmids and virus. The human IL-2 gene was amplified by PCR using Primers containing SpeI or BsiWI sites (5'-gcAC TAGTacaggatgcaactcctgtcttgc-3' and 5'-gaCGTACGattaagt cagtgttgagatgatgc-3') and the Expand ${ }^{\mathrm{TM}}$ Long Template PCR System kit (Boehringer-Mannheim, Germany) from the plasmid pSVT7-hIL2 (obtained from Dr M. Zöller, DKFZ, Heidelberg, Germany). The obtained fragment was then cloned into the pGEM-T vector resulting in pGEM-T-hIL-2, which was sequenced to confirm that no mutation has occurred. The SpeI and BsiWI fragment (759 bp) of this plasmid was then subcloned into the SpeI and BsiWI sites of a modified pUC19 plasmid that contained an oligonucleotide bearing the recognition sequences for SpeI and BsiWI (5'-AATTACTA GTGGCCCGTACGC-3'), which was synthesized and cloned into the EcoRI site of pUC19. This vector including the SpeIBsiWI fragment was applied to replace the corresponding fragment in pNDFL (1). In a second step, an additional transcriptional unit including the NDV gene start, gene end signals and hIL-2 open reading frame were inserted into the full length NDV genome. The integrity of the entire foreign sequence was confirmed by sequencing. The resulting plasmid was named pNDFL-hIL-2 (13). Construction and rescue of the rec(IL-2) was performed according to $\operatorname{rec}(\mathrm{GM}-\mathrm{CSF})$ described in Janke et al (14). All the viruses (recombinant or not) were propagated in embryonated chicken eggs, harvested from the allantoic fluid, purified by ultracentrifugation and quantified by hemagglutination as described previously (12).

Purification of blood derived cells. Human peripheral blood mononuclear cells (PBMC) were isolated from buffy coat of healthy donors by Ficoll-Paque (Amersham Pharmacia Biotech, Freiburg, Germany) density gradient centrifugation. $\mathrm{T}$ cells were purified from PBMC by magnetic bead separation via antibody-coated Dynabeads using a $\mathrm{T}$ cell negative isolation kit containing mAbs against CD14, CD16 (a and b), CD56, HLA Class II DR/DP and beads coated with an Fc-specific human IgG4 against mouse IgG (Dynal, Hamburg, Germany) or using anti-CD3 MACS-microbeads (Miltenyi, Bergisch Gladbach, Germany) following the manufacturer's guidelines.

Isolation of patient derived autologous T-lymphocytes. T cells autologous to HNSCC patient derived tumor cells were isolated from lymph nodes of these patients and incubated for 7 days in RPMI-1640 (Invitrogen) supplemented with 10\% human AB serum, 2\% autologous plasma, IL-2 (100 U/ml), and IL-4 (60 U/ml) (all PromoCell) followed by overnight incubation 
in medium without cytokines and magnetic bead depletion (Dynal, Hamburg, Germany) of contaminating CD19+ B-cells, CD15 ${ }^{+}$myeloid cells, CD56 ${ }^{+}$NK-cells, and NK-T-cells. Cells were stored in liquid nitrogen until use.

Production of virus infected tumor vaccine stimulator cells. As stimulator cells for mixed lymphocyte-tumor cell cultures (MLTC) and for the tumor neutralization assay (TNA), we used MCF-7 breast carcinoma cells that were inactivated by 200 Gy irradiation via a ${ }^{137} \mathrm{Cs}$ source (Gammacell 1000, Atomic Energy of Canada, Chalk River, Canada) and then infected with the respective NDV strain (100 HU per $10^{7}$ cells per ml) as described (15).

Cytokine-dependent proliferation assay. We used IL-2dependent CTLL cells to analyze the biological activity of the expressed integrated cytokine gene released during viral tumor cell infection. One day prior to the assay, the indicator cells were transferred to cytokine-free medium. For the assay, the cells were harvested, washed three times with cytokine-free medium and adjusted to $2 \times 10^{5} / \mathrm{ml}$. Fifty $\mu 1$ of cell suspension were added to a 96-well plate, which contained serial 1:4 dilutions of the supernatants of infected tumor cells. The assay was performed in duplicates for each dilution step. After $48-72 \mathrm{~h}$ at $37^{\circ} \mathrm{C} 1 \mu \mathrm{Ci}\left[{ }^{3} \mathrm{H}\right]$-thymidine in $50 \mu \mathrm{l} \mathrm{RPMI}$ with $5 \%$ FCS was added to each well of the plate, which then was incubated for additional $4 \mathrm{~h}$ at $37^{\circ} \mathrm{C}$. By using a micro-cell harvester, the radioactive DNA was transferred onto a fiber glass mat, which was analyzed in a liquid scintillation counter (Perkin-Elmer Wallac, Freiburg, Germany) for thymidine incorporation.

Flow cytometry. A total of $5-10 \times 10^{5}$ cells were used per sample. All antibodies were diluted in FACS buffer (PBS containing $5 \% \mathrm{FCS}$ and $0.1 \% \mathrm{NaN}_{3}$ ). Cells were incubated with a primary (un-)conjugated antibody for $20 \mathrm{~min}$ on ice in the dark. Subsequently the cells were washed two times with FACS buffer and, if necessary, incubated with a conjugated secondary antibody for $20 \mathrm{~min}$ on ice in the dark. Then the cells were washed two times, adjusted to a volume of $100 \mu 1$ and analyzed in a FACSCalibur or LSR II flow cytometer (Becton Dickinson, Heidelberg, Germany). Intracellular staining was performed using the Cytoperm/Cytofix kit (BD, Heidelberg, Germany) in accordance to the manufacturer's guidelines. All FACS data were analyzed with CellQuest Pro software (Becton-Dickinson) or Flow Jo software (Tree Star Inc., Ashland, USA).

IFN- $\gamma$ ELISPOT assay. IFN- $\gamma$ producing T lymphocytes were determined using an IFN- $\gamma$ ELISPOT Kit (Mabtech, Hamburg, Germany). T cells $\left(2 \times 10^{5}\right)$ isolated from HNSCC tumor patients were incubated in a 96-well ELISPOT plate with $2 \times 10^{4}$ autologous irradiated tumor cells or U937 cells infected with the respective NDV strain and loaded with $0.15 \mu \mathrm{g}$ bispecfic antibodies or not. For negative control untreated $\mathrm{T}$ cells or $\mathrm{T}$ cells co-incubated with uninfected irradiated tumor cells were used. After $40 \mathrm{~h}$ the plates were analyzed in accordance to the manufacturer's guidelines of the ELISPOT kit using the ELISPOT substrate kit of Bio-Rad Labs (Hercules, USA). The number of IFN- $\gamma$ spots was measured automatically with KS ELISPOT software (Carl Zeiss Vision, Hallbergmoos, Germany). The frequency of tumor-reactive T cells is shown as IFN- $\gamma$ producing cells per $2 \times 10^{5} \mathrm{~T}$ cells.

Tumor neutralization assay (TNA). The assay was performed in 48-well round-bottom plates as previously described (14). Briefly $1.4 \times 10^{4}$ live MCF-7 tumor cells were added in DMEM with $5 \%$ FCS to each well and incubated for $4 \mathrm{~h}$ at $37^{\circ} \mathrm{C}$ to adhere. Subsequently $9.6 \times 10^{4}$ freshly isolated non-activated $\mathrm{T}$ cells from normal healthy donors were added to the respective wells. $2.4 \times 10^{4} \mathrm{MCF}-7$ tumor vaccine stimulator cells per well were prepared by irradiation and virus infection as described. The micro-titer plates were incubated in a $\mathrm{CO}_{2}$ incubator at $37^{\circ} \mathrm{C}$. At the end of the assay, supernatants were removed by suction and the plates were carefully washed. Then $200 \mu 120 \%$ MTS solution were added per well and incubated for $20-40 \mathrm{~min}$ at $37^{\circ} \mathrm{C}$ in the dark. The reaction was stopped by adding $10 \%$ SDS solution. The plates were then evaluated in an ELISA reader at $490 \mathrm{~nm}$. The background was taken from control wells containing only medium and MTS solution. The result was displayed as percent tumor growth inhibition related to an untreated tumor cell monolayer (positive control) calculated according to:

$1-\left(\frac{\mathrm{A}_{490} \text { experimental well }-\mathrm{A}_{490} \text { background }}{\mathrm{A}_{490} \text { positive control }-\mathrm{A}_{490} \text { background }}\right) \times 100=\%$ growth inhibition

Cytotoxicity assay. Purified T cells were stimulated with rec(-) and rec(IL-2) infected, irradiated MCF-7 tumor cells at a ratio of $4: 1$ for 6 days. All cells were recovered from the culture, counted and used as effector cells. MCF-7 tumor cells $\left(2 \times 10^{6}\right)$ were labeled with $200 \mu \mathrm{Ci}$ of ${ }^{51} \mathrm{Cr}$ for $1 \mathrm{~h}$ at $37^{\circ} \mathrm{C}$, washed four times, and resuspended at $2 \times 10^{5}$ cells $/ \mathrm{ml}$ medium. To quantify $\mathrm{T}$ cell induced cell death, ${ }^{51} \mathrm{Cr}$-labeled tumor cells $\left(10^{4}\right.$ cells/well $)$ were incubated with varying numbers of T cells for $4 \mathrm{~h}$. The assay (15) was performed in 96-well round-bottom plates in triplicate, and the percent specific lysis was calculated after analysis of radioactivity in the supernatants of the wells as: $100 \mathrm{x}$ (experimental cpm spontaneous cpm)/(total cpm - spontaneous cpm). Spontaneous and total ${ }^{51} \mathrm{Cr}$ release values were determined in the presence of either medium alone or $10 \%$ Triton X-100.

\section{Results}

Binding and replication capacities of rec $(I L-2)$ virus. We describe a new recombinant (rec) NDV virus with an integrated gene coding for human IL-2. The gene was incorporated in front of the viral NP gene as described for rec(GM-CSF) virus (14). This rec(IL-2) virus can be used to prepare a tumor vaccine that produces IL-2 at the vaccination site aiming at local improvement of adaptive $\mathrm{T}$ cell mediated immune responses compared to systemic IL-2 application.

For production of a cell based tumor vaccine and for comparison with other viruses it is important to know tumor cell binding and virus replication properties. To this end we infected the human breast carcinoma cell line MCF-7 with rec(-) or rec(IL-2) virus or with the wild-type (wt) virus of strain Ulster and analyzed the expression of the viral fusion protein $\mathrm{F}$ at the cell surface after $1 \mathrm{~h}$ (for virus binding) and after $24 \mathrm{~h}$ (for replication properties). 
wt (Ulster)
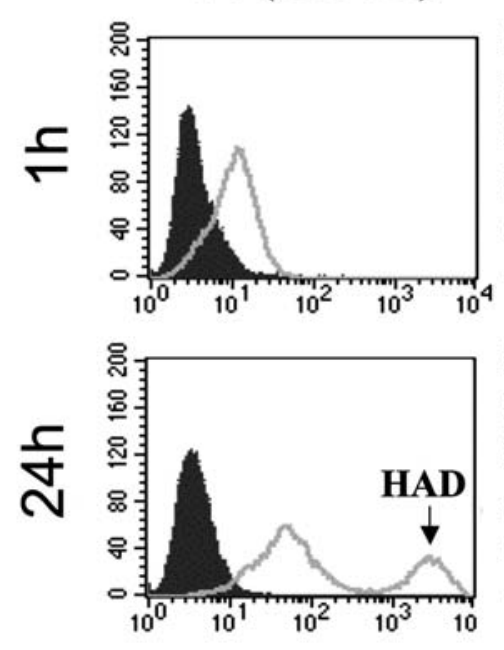

$\operatorname{rec}(-)$
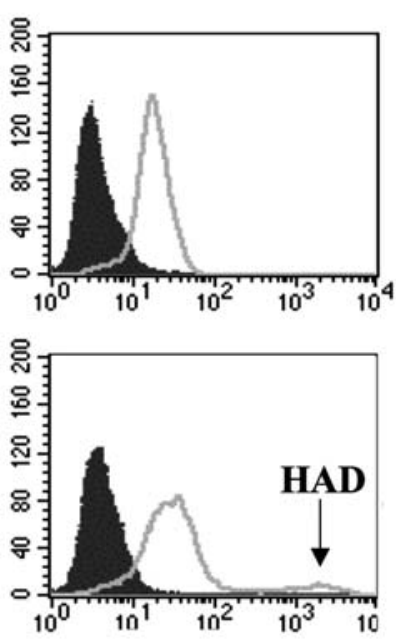

$\operatorname{rec}(\mathrm{IL}-2)$
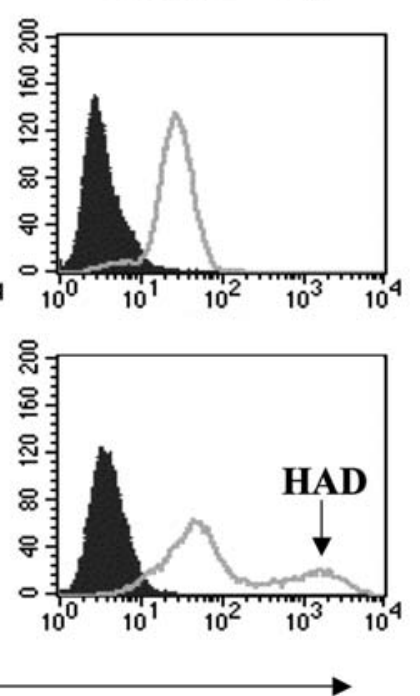

NDV fusion protein (F)

Figure 1. Tumor cell binding and virus replication of the recombinant NDV strains. MCF-7 cells $\left(1 \times 10^{7}\right)$ were incubated in $1 \mathrm{ml}$ volume with $100 \mathrm{HU}$ of the indicated NDV strains for $1 \mathrm{~h}$ at $37{ }^{\circ} \mathrm{C}$ and then washed. Virus binding (A) and replication (B) were analyzed by staining for cell surface expressed viral $\mathrm{F}$ protein directly after infection (top panel) or after further incubation for $24 \mathrm{~h}$ to allow for virus replication (bottom panel). Staining was performed with mouse anti-F mAb (1 $\mu \mathrm{g} / 5 \times 10^{5}$ cells) followed by PE-labeled goat anti-mouse secondary antibody (1:50). Dark profiles show staining controls with secondary antibody only. White histograms show the expression of viral F protein at indicated time-points. Note that without viral replication there is only F-expression at low antigen density whereas after infection and replication the tumor cells show a distinct population of cells with F-expression at high antigen density (HAD). One representative experiment out of five is shown. NDV, Newcastle disease virus; IL-2, interleukin 2.

Fig. 1 shows that the rec virus strains derived from lentogenic NDV La Sota behaved similar to the previously used lentogenic NDV Ulster (3). Viral replication in the cytoplasm was associated with an increase of the density of cell surface expressed viral $\mathrm{HN}$ and F proteins. Similar results were obtained with other tumor cell lines like MCF-10A, HT-29 colon carcinoma or Jurkat lymphoma (data not shown). The rec strains were also similar to NDV Ulster in that they were not able to replicate in normal human cells (10) and that supernatants from infected tumor cells were non-infectious (16) due to an uncleaved F protein in the newly synthesized virion particles (data not shown) (17).

Production of IL-2 and its biological activity. As shown in Fig. 2A, human MCF-7 breast carcinoma cells infected with rec(IL-2) virus produced $\sim 40 \mathrm{ng}$ of IL-2 within the first $24 \mathrm{~h}$. Maximum levels of cytokine expression were observed after $48 \mathrm{~h}$ of cell culture and this level remained constant at least until 96 h of culture. No IL-2 protein could be detected in the supernatant of tumor cells infected with rec(-) virus and $\gamma$-irradiation had no influence on protein expression (data not shown). Fig. 2B shows that the expression of the therapeutic gene product was strictly dependent on the number of irradiated MCF-7 vaccine cells added per well of a 96well plate. While $1500 \gamma$-irradiated infected vaccine cells produced only $6.6 \mathrm{ng} / \mathrm{ml}, 96,000$ cells produced $127 \mathrm{ng} / \mathrm{ml}$ IL-2 within $48 \mathrm{~h}$ of cell culture.

The produced IL-2 in the supernatant of rec(IL-2) infected MCF-7 cells had the capacity to stimulate the growth of the IL-2 dependent cell line CTLL in a linear dose-dependent fashion (Fig. 2C), whereas supernatants of rec(-) infected tumor cells failed to induce proliferation. The produced IL-2 was reasonably stable at $37^{\circ} \mathrm{C}$ for at least 16 days with an average degradation rate of $25 \%$ during this time period (data not shown). In conclusion rec(IL-2) infected human tumor cells produce IL-2 as a stable gene product with biological activity.

Effect of a rec(IL-2) infected tumor cell vaccine in a tumor neutralization assay with purified T cells. The TNA is basically a mixed lymphocyte-tumor cell culture (MLTC) performed on top of a tumor cell monolayer so that cytotoxic and bystander anti-tumor effects can be measured directly. 14,000 MCF-7 human breast carcinoma cells were transferred per well of 48-well plates and incubated for $4 \mathrm{~h}$ to enable their adherence. Then 96,000 purified T cells from blood of healthy donors were added as effector cells (E). As vaccine (V) we added 24,000 MCF-7 cells, which had been inactivated by $\gamma$-irradiation and infected by either rec(-) or rec(IL-2) virus. Each well contained a total of $400 \mu 1$ culture medium. The plates were incubated for 6 days at $37^{\circ} \mathrm{C}$, washed and the remaining adherent cells were stained with the vital dye MTS. Fig. 3 shows a summary of 13 different TNAexperiments performed with $\mathrm{T}$ cells from 13 different healthy donors. While in absence of virus infection, the vaccine cells did not induce tumor growth inhibition (TGI), 26-29\% of TGI was observed when virus modified vaccine cells were added on top of the tumor cell monolayer. Upon addition of $\mathrm{T}$ cells we observed an increase of TGI in groups containing virus infected vaccine cells. The increase depended on the type of virus used for vaccine modification. The strongest effect was observed with T cells stimulated with V[rec (IL-2)]. 
A

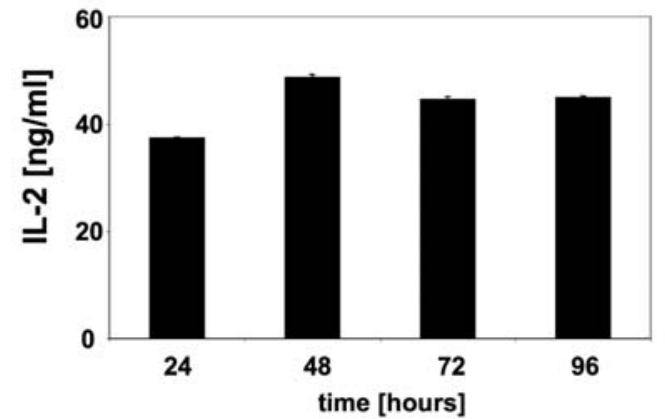

B
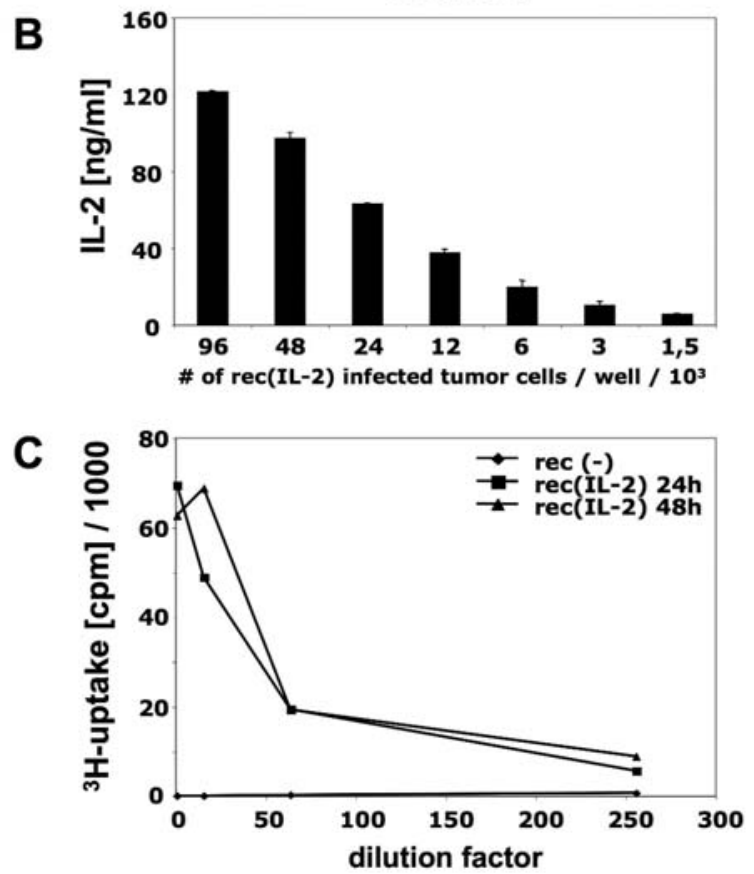

Figure 2. Expression of the therapeutic gene product in tumor cells after infection with rec NDV. (A) Kinetics of the expression of the therapeutic gene product. MCF-7 tumor cells were irradiated with $200 \mathrm{~Gy}$ as for vaccine production, infected with rec(IL-2) and cultured as described for Fig. 1. Twenty-four, 48, 72 and 96 hours later supernatants were tested for content of IL-2 by ELISA. rec(-)-infected control cultures did not produce IL-2 (data not shown). (B) Dependence of the expression of the therapeutic gene product on the number of irradiated, infected MCF-7 vaccine cells. After infection with rec(IL-2), MCF-7 cells were seeded at different cell numbers per well (1500-96000 per $200 \mu 1)$ in a 96-well plate. The expression of the therapeutic gene product was analyzed $48 \mathrm{~h}$ later by ELISA. The columns show means and standard deviations from triplicates of one representative experiment. (C) Biological activity of the produced IL-2 protein. MCF-7 cells were infected with rec(-) or rec(IL-2) and cultured at $1.4 \times 10^{5}$ cells per $\mathrm{ml}$ for 24 and $48 \mathrm{~h}$. Supernatants of these cultures were added in serial 1:4 dilutions to IL-2 sensitive CTLL cells, incubated for $72 \mathrm{~h}$ and the proliferation of these cells was determined by measuring uptake of ${ }^{3} \mathrm{H}$ labeled thymidine during a 4-h pulse. NDV, Newcastle disease virus; ELISA, enzyme-linked immunosorbent assay; IL-2, interleukin 2.

The TGI of this group was significantly higher than in the group of T cells stimulated with V[rec(-)], thus demonstrating the added value of the transgene in the virus.

Experiments with $\mathrm{T}$ cells stimulated with $\mathrm{V}[\mathrm{rec}(-)]$ and recombinant IL-2 mimiking V[rec(IL-2)] show that the IL-2 expressed by rec(IL-2) infected tumor cells is most likely the factor that leads to the increased anti-tumor effect since $\mathrm{V}[\mathrm{rec}(-)]$ and recombinant IL-2 also leads to a significant increase of TGI compared to $\mathrm{V}[\mathrm{rec}(-)]$ stimulation alone (data not shown).

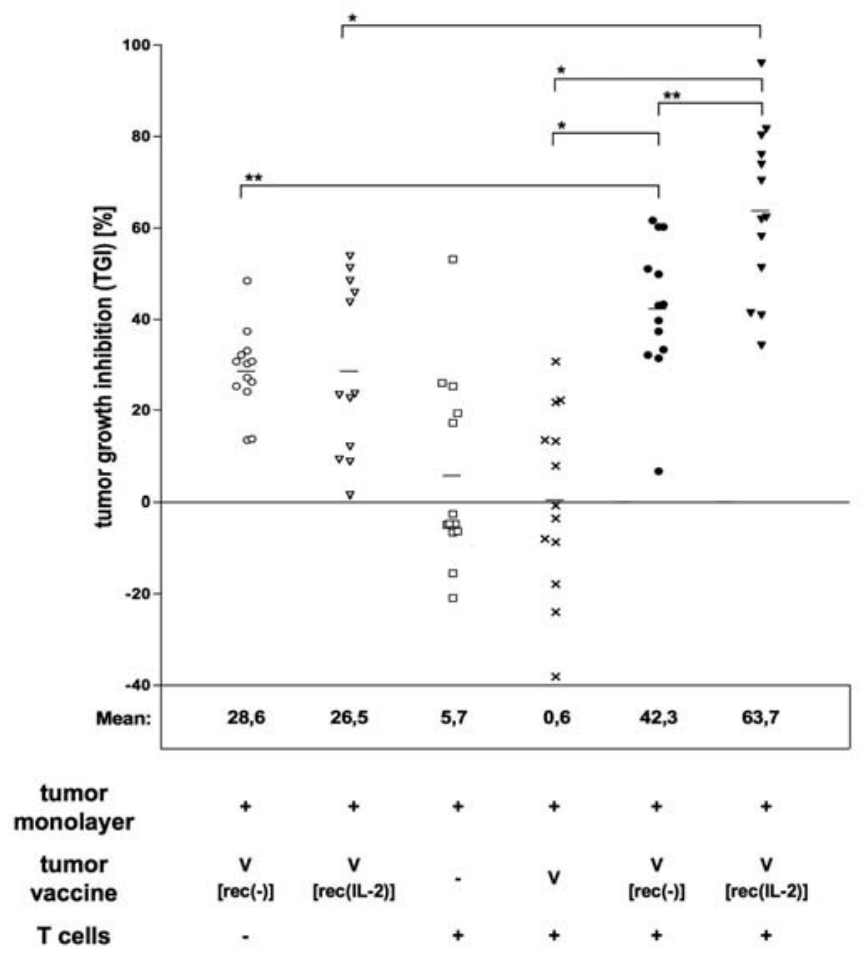

Figure 3. Effect of rec(IL-2) infected tumor cells on T cell mediated tumor growth inhibition in a tumor neutralization assay. Dynabead purified T cells of healthy donors were co-cultured with irradiated, non-infected (V), rec(-) or rec(IL-2) infected MCF-7 tumor cells (V[rec(-)], V[rec(IL-2)]) on top of a live MCF-7 tumor cell monolayer. Time of incubation depended on confluence of an untreated monolayer control. T cells and stimulator cells were removed by suction of the supernatant. Monolayers were stained with $20 \%$ MTS solution diluted in RPMI-medium supplemented with 5\% FCS for $20-40 \mathrm{~min}$ at $37^{\circ} \mathrm{C}$ in the dark. Results were evaluated in a multiplate reader at $490 \mathrm{~nm}$ and displayed as \% of tumor growth inhibition (\% TGI) calculated according to Materials and methods. Figure shows the summary of 13 independent experiments showing each single donor and the mean of each group. ${ }^{*} \mathrm{p}<0.001 ;{ }^{* *} \mathrm{p}<0.003$; TGI, tumor growth inhibition; IL-2, interleukin 2.

Upregulation of $T$ cell activation markers and expression of $I F N-\gamma$. Since we observed increased anti-tumor effects when using $\mathrm{T}$ cells stimulated with rec(IL-2) infected tumor vaccine we next evaluated the activation status of the $\mathrm{T}$ cells. We analyzed cell surface expression of the early activation marker CD69 and release of the T cell cytokine IFN- $\gamma$. We performed TNA assays and after 3, 4 and 5 days the T cells were removed from the culture and stained with differently labeled monoclonal antibodies (mAbs) against CD3, CD4, CD8 and CD69. The results of CD69 expression from three experiments expressed by mean fluorescence intensity (MFI) for $\mathrm{CD}_{3}{ }^{+} \mathrm{CD}^{+}$and $\mathrm{CD}^{+}{ }^{+} \mathrm{CD} 8^{+} \mathrm{T}$ cells are shown in Fig. 4A. A slight increase in CD69 expression was seen for T cells stimulated with rec(-) infected vaccine compared to $\mathrm{T}$ cells that were stimulated with uninfected vaccine $(\mathrm{V})$. A strong effect was only obvious when the $\mathrm{T}$ cells were stimulated with MCF-7 vaccine infected with rec(IL-2). In this case, CD69 upregulation was significantly higher than in the $\mathrm{V}[\mathrm{rec}(-)]$ stimulated group. This was true over the whole time course and for both $\mathrm{CD}^{+}$and $\mathrm{CD} 8^{+} \mathrm{T}$ cells.

Supernatants from TNA cultures were further investigated by ELISA assays for content of interferon- $\gamma$. The results are 


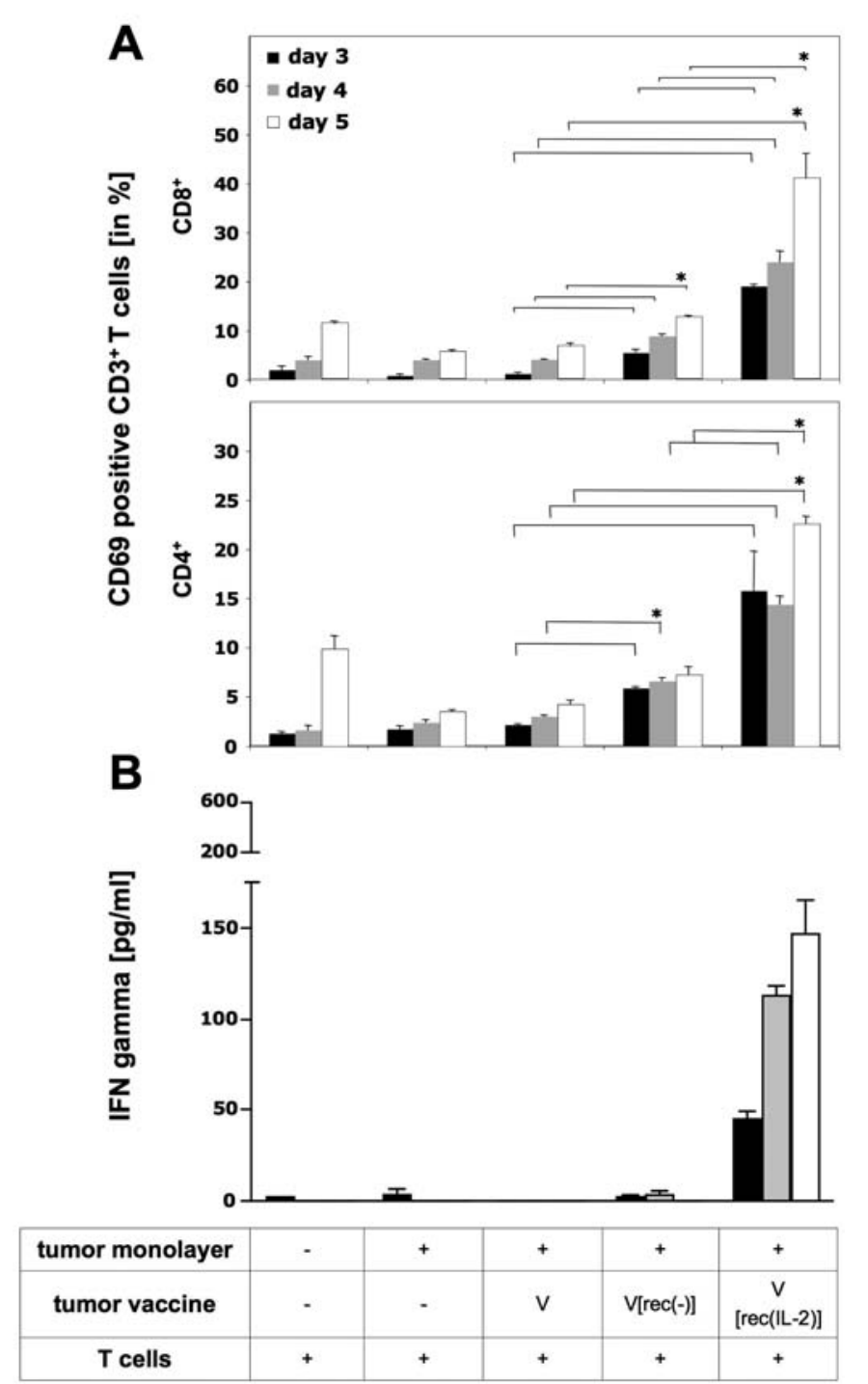

Figure 4. Activation of $\mathrm{T}$ cells stimulated with rec(IL-2) infected tumor cells. MACS purified T cells of healthy donors were co-cultured with irradiated, non-infected (V), rec(-) or rec(IL-2) infected MCF-7 tumor cells (V[rec(-)], V[rec(IL-2)]) on top of a live MCF-7 tumor cell monolayer for different time periods. Data show the mean of three independent experiments with SEM. (A) CD69 expression on gated T cell populations. Cells were harvested, stained with fluorescence labeled monoclonal antibodies against CD3, CD4, CD8 and CD69 and analyzed by flow cytometry. Data are presented as percentage of $\mathrm{CD} 69$ positive $\mathrm{CD} 3^{+} \mathrm{CD} 8^{+} \mathrm{T}$ cells (top panel) or $\mathrm{CD}^{+}{ }^{+} \mathrm{CD} 4{ }^{+} \mathrm{T}$ cells (bottom panel). ${ }^{*} \mathrm{p}<0.05$. (B) Supernatants were taken and analyzed for IFN- $\gamma$ expression by ELISA. " $\mathrm{p}<0.01$; ELISA, enzymelinked immunosorbent assay; IL-2, interleukin 2; rec, recombinant.

shown in Fig. 4B. Supernatants of cultures in which purified $\mathrm{T}$ cells were stimulated with a vaccine infected by rec(IL-2) contained significantly higher amounts of IFN- $\gamma$ in comparison to the other cultures. The induced IFN- $\gamma$ as well as the CD69 induction could be mimicked by rec(-) infected tumor cells and exogenous IL-2 (data not shown). Thus, rec(IL-2) infected vaccine cells had an increased capacity to stimulate $\mathrm{T}$ cells to produce IFN- $\gamma$ and to exert anti-tumor effects.

Functional activity of $T$ cells stimulated with rec(IL-2) infected tumor cells. Next we tested the functionality of the stimulated $\mathrm{T}$ cells. First we investigated the intracellular expression of perforin in activated CD8 $\mathrm{T}$ cells. The results are shown in Fig. 5A. About $9 \%$ of $\mathrm{CD}^{+} \mathrm{CD}^{+} \mathrm{T}$ cells activated with vaccine cells infected with rec(IL-2) had upregulated perforin expression (4\% also expressed CD69) in comparison to $1 \%$ of $\mathrm{T}$ cells activated with vaccine infected with rec(-) $(0.1 \%$ also expressed CD69). Since cytotoxic CD8 T cells fuse intracellular cytotoxic granules with their outer membrane, thereby expressing the marker CD107a (18), we performed further TNA experiments to analyze the expression of this intracellular vesicle marker on the T cell surface. We stained the T cells from TNA assays for CD3, CD4, CD8, CD69 and CD107a. Unstimulated T cells showed only intracellular expression of CD107a but no surface expression (data not shown). Fig. 5B shows cell surface exposed CD107a only on $\mathrm{T}$ cells stimulated with rec(IL-2) infected tumor cells and not on $\mathrm{T}$ cells stimulated with rec(-) infected tumor cells. There was much less CD107a expression on CD4 cells (data not shown). This is in accordance with the known function of this molecule in relation to the exocytosis and release of cytotoxic molecules by CD8 cytotoxic T cells. The percentage of $\mathrm{CD} 107$ a positive $\mathrm{CD}^{+} \mathrm{T}$ cells stimulated with rec(IL-2) infected tumor cells increased with time reaching about $30 \%$ after 5 days (Fig. 5C).

In order to confirm that rec(IL-2) infected tumor cells can induce true cytotoxic T lymphocytes (CTLs) we performed a ${ }^{51} \mathrm{Cr}$-release assay with MCF-7 target cells. The results are shown in Fig. 5D. CTLs stimulated with rec(IL-2) infected MCF-7 vaccine cells had a higher cytotoxic activity than unstimulated cells or cells stimulated with rec(-) infected vaccine.

Stimulation of cancer patient's T cells with autologous tumor cells infected with rec(IL-2). We previously demonstrated the presence of tumor reactive memory $\mathrm{T}$ cells in patients with different types of cancer (19). By means of the ELISPOT assay we were able to quantify the number of $\mathrm{T}$ cells that react by interferon- $\gamma$ secretion upon stimulation with autologous dendritic cells (DCs) loaded with tumor lysate or defined tumor associated antigens (20). We also obtained positive results from phase-II clinical studies in which the autologous virus modified tumor vaccine ATV-NDV was employed as a vaccine (3). It was therefore of interest to find out whether an autologous tumor vaccine modified by infection with the IL-2 producing virus could stimulate cancer reactive memory $\mathrm{T}$ cells from patients ex vivo. Since one of the positive vaccination studies was performed in head and neck squamous cell carcinoma patients (6), we selected such patients from whom we could obtain lymph nodes for the isolation of $\mathrm{T}$ cells and generated autologous tumor cell lines. The T cells were stimulated on top of an ELISPOT membrane coated with anti-interferon $-\gamma$ mAb with unmodified or modified autologous tumor cells for $40 \mathrm{~h}$. After washing, the plates were developed with biotinylated anti-interferon- $\gamma \mathrm{mAb}$ of a different specificity and enzyme labeled streptavidin. Interferon- $\gamma$ producing $\mathrm{T}$ cells were made visible as blue spots upon substrate addition. For virus-infection of the vaccine we either used rec(-) or rec(IL-2). Fig. 6A shows the quantitative evaluation of the reactivities from four patients. While $\mathrm{T}$ cells cultured alone produced no spots, upon coincubation with rec(-) infected vaccine a mean of 2 spots per 

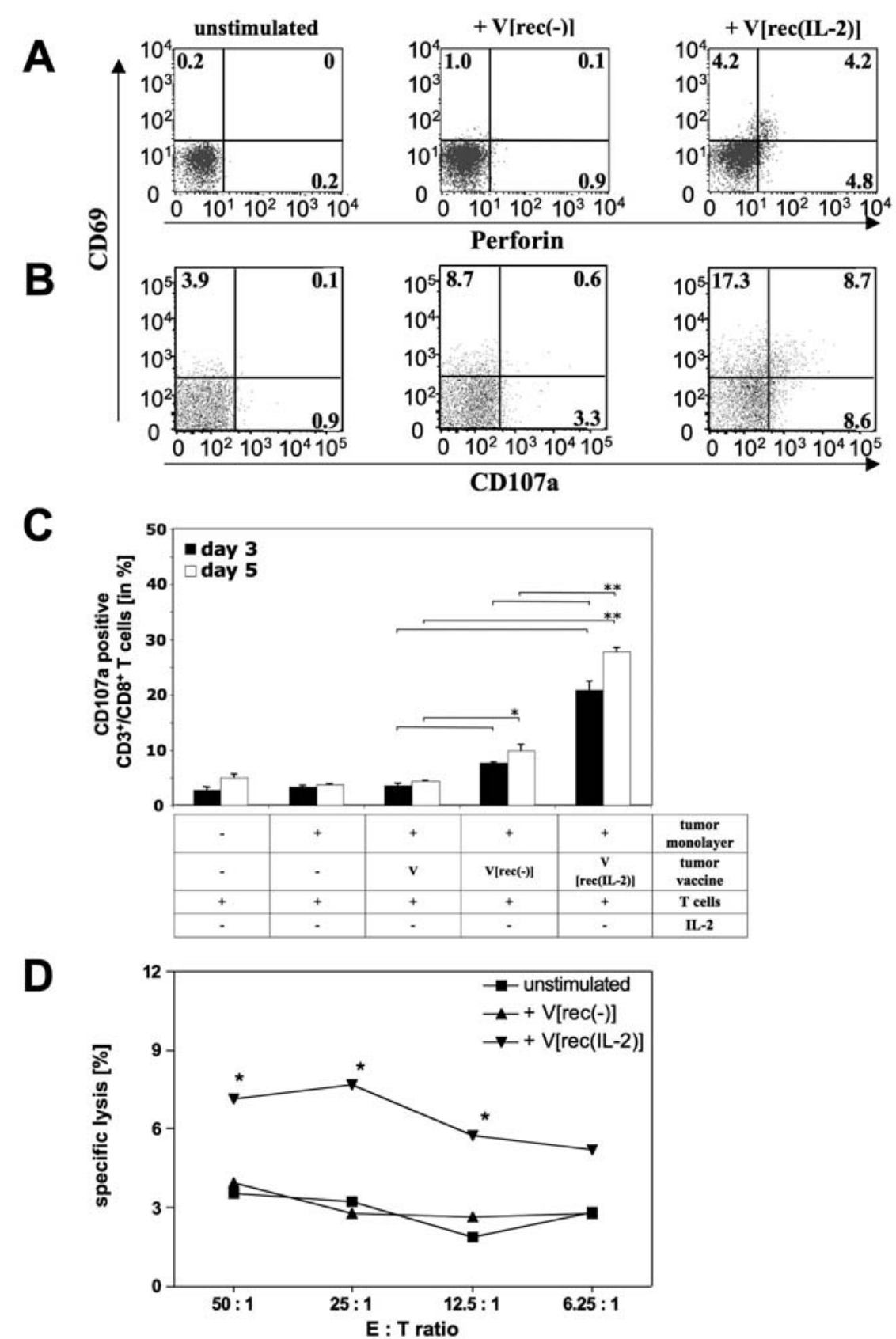

Figure 5. Induction of T cell cytotoxicity after stimulation with rec(IL-2) infected tumor cells. Purified T cells of healthy donors were co-cultured with irradiated, non-infected (V), rec(-) or rec(IL-2) infected MCF-7 tumor cells (V[rec(-)], V[rec(IL-2)]) on top of a live MCF-7 tumor cell monolayer for different time periods. (A and B) Expression of cytotoxicity markers on CD8 T cells after stimulation with rec(IL-2) infected tumor cells. After day 4 cells were harvested, stained with fluorescence labeled monoclonal antibodies against CD3, CD8 and CD69 and analyzed by flow cytometry. (A) After fixation and permeabilization, cells were stained with a monoclonal antibody against perforin and analyzed by flow cytometry. Dot plots show expression of CD69 and perforin on gated $\mathrm{CD}^{+} \mathrm{CD}^{+} \mathrm{T}$ cells. Numbers indicate percentages of positive cells. One of two experiments is shown. (B) Cells were additionally stained with monoclonal antibodies against CD107a. Numbers indicate percentages of positive $\mathrm{CD}^{+} \mathrm{CD}^{+} \mathrm{T}$ cells. One representative experiment of 4 is shown. (C) Summary of three independent experiments for CD107a expression after days 3 and 5. Data show the mean with SEM. ${ }^{*}<<0.03$; ${ }^{* *}$ p $<0.004$. (D) Induction of cytotoxicity after $\mathrm{T}$ cell stimulation with rec(IL-2) infected tumor cells. $\mathrm{MCF}-7$ tumor cells were labeled with $200 \mu \mathrm{Ci}$ of ${ }^{51} \mathrm{Cr}$ for $1 \mathrm{~h}$ and resuspended at $2 \times 10^{5}$ cells $/ \mathrm{ml}$. T cells were recovered from a 6-day 4:1 ratio culture of Dynabead isolated T cells and irradiated rec(-) or rec(IL-2) infected MCF-7 cells. ${ }^{51} \mathrm{Cr}$-labeled tumor cells $\left(10^{4}\right.$ cells/well $)$ were incubated in triplicates with varying numbers of recovered $\mathrm{T}$ cells yielding E:T ratios from 50:1 to 6.25:1 for $4 \mathrm{~h}$. The percentage of specific lysis was calculated after analysis of radioactivity in the supernatants of the wells. Data represent mean of three experiments. ${ }^{*} \mathrm{p}<0.02$; IL-2, interleukin 2 ; rec, recombinant.

$2 \times 10^{5} \mathrm{~T}$ cells was observed. This was increased to 8 when rec(IL-2) infected vaccine cells were used.

To increase the $\mathrm{T}$ cell co-stimulatory activity of the autologous virus modified tumor vaccine we modified it further by attachment of an anti-HN/anti-CD28 bispecific single- chain antibody construct (bsHN-CD28) (21). In this group we saw a further increase to 44 spots per $2 \times 10^{5} \mathrm{~T}$ cells. Because of the short incubation time of the assay (18) it is likely that the spot forming cells represent mostly memory $\mathrm{T}$ cells rather than naïve T cells. Further addition of a bispecific anti- 
A

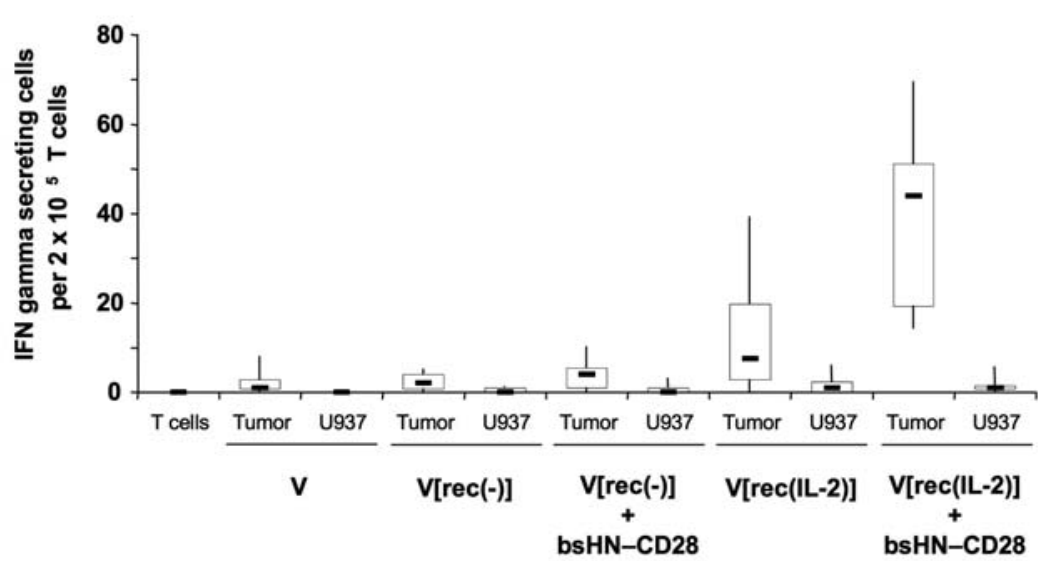

B

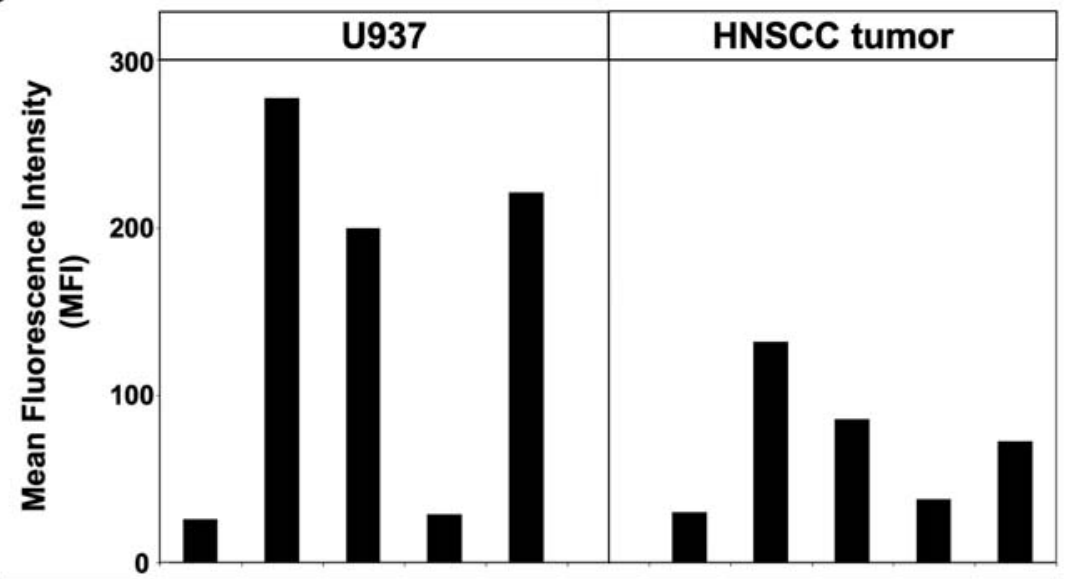

\begin{tabular}{|c|c|c|c|c|c|c|c|c|c|c|c|}
\hline anti-HN & - & + & - & - & - & & - & + & - & - & - \\
\hline anti-F & - & - & + & - & - & & - & - & + & - & - \\
\hline $\begin{array}{c}\text { anti-HN- } \\
\text { CD28 }\end{array}$ & - & - & - & - & + & & - & - & - & - & + \\
\hline anti-Flag & - & - & - & + & + & & - & - & - & + & + \\
\hline GaM-PE & + & + & + & + & + & & + & + & + & + & + \\
\hline
\end{tabular}

Figure 6. Stimulation of T cells by rec(IL-2) infection in an autologous tumor model. Tumor cells and autologous T cells of four different HNSCC cancer patients were isolated and cultured as described in Materials and methods. Autologous tumor cells and U937 cells for control were irradiated and infected with rec(-) or rec(IL-2) as described. Twenty-four hours after infection cells were recovered from culture. In some samples a Flag-tagged bispecific antibody was used binding viral HN-protein and CD28 (bsHN-CD28) and leading to co-stimulation of T cells. (A) IFN- $\gamma$ production of autologous T cells after stimulation with infected tumor cells. Isolated T cells $\left(2 \times 10^{5}\right)$ were incubated in a 96-well ELISPOT plate with $2 \times 10^{4}$ autologous irradiated and infected tumor or U937 cells loaded with $0.15 \mu \mathrm{g}$ of the bispecfic construct bsHN-CD28 or not. For negative control untreated T cells or T cells co-incubated with uninfected irradiated tumor cells were used. After $40 \mathrm{~h}$ the plates were analyzed and the number of IFN- $\gamma$ spots was measured automatically with KS ELISPOT software. Each box plot column represents the pooled median, $25 \%$ and $75 \%$ (bars $5 \%$ and $95 \%$ ) quartile counts of IFN- $\gamma$-secreting tumor reactive T cells per $2 \times 10^{5}$ total $\mathrm{T}$ cells from the four different patients. (B) Expression of viral antigens and binding of the bispecific co-stimulatory construct. Infected HNSCC tumor cells and U937 cells were stained with mouse anti-F-, mouse anti-HN- or mouse anti-Flag-mAb (all $1 \mu \mathrm{g} / 5 \times 10^{5}$ cells) followed by PE-labeled goat anti-mouse secondary antibody (1:50). The group containing the bispecific construct was incubated with bsHN-CD28 ( $0.6 \mu \mathrm{g} / 5 \times 10^{5}$ cells) for $30 \mathrm{~min}$ before staining. Cells were analyzed by flow cytometry. Data are shown as mean fluorescence intensity (MFI). IL-2, interleukin 2.

HN/anti-CD3 protein $(15,21)$ to the same T cells caused a significant increase of the mean spot number to 174 ( $p<0.06$, data not shown).

For specificity control we included a heterologous tumor vaccine unrelated to HNSCC tumors. It consisted of the pro-myelomonocytic cell line U937. This was modified in exactly the same way as the four autologous HNSCC tumor lines. Interestingly, the patient derived $\mathrm{T}$ cells did not react to the heterologous U937 vaccine, no matter how it was modified (Fig. 6B). These results support our assumption that we are testing an anti-tumor memory $\mathrm{T}$ cell response in this assay. It was important, however, to exclude other reasons for the difference, e.g. difference in: i) virus infectivity; or in: ii) binding of the bispecific proteins. Fig. 6B shows a FACS analysis of U937 and a HNSCC tumor cell line after infection with rec(-) and binding of the bispecific protein bsHN-CD28. The results reveal that U937 cells could be even better infected by the virus and could bind more of the bispecific proteins than the autologous cell line. The extent of virus infection is measured by staining with NDV specific anti-HN and anti-F-mAb, while the binding of the bsHN$\mathrm{CD} 28$ construct is revealed by staining with anti-Flag $\mathrm{mAb}$ 
thereby detecting the Flag-tag of this recombinant protein. U937 cells also showed no abnormal expression of HLA class I, and they were negative for HLA-class II expression (data not shown). In conclusion, testing the response of lymph node $\mathrm{T}$ cells from HNSCC cancer patients revealed that only the stimulation with rec(IL-2) modified autologous tumor cells was able to generate short-term ELISPOT responses.

\section{Discussion}

This report describes a new recombinant NDV strain with an incorporated therapeutic gene. Upon infection of tumor cells, this new agent replicates and produces the therapeutic gene product IL-2, which is secreted in large amounts into the supernatant. When such a vaccine is used in a mixed lymphocyte tumor-cell culture (MLTC) to stimulate human T cells, some of the $\mathrm{T}$ cells become activated, express CD69 and secrete the $T$ cell cytokine interferon $-\gamma$. A fraction of CD8 $\mathrm{T}$ cells showed upregulated expression of perforin and transported intracellular cytotoxic granules to their outer membrane thereby exposing CD107a. T cells stimulated with rec(IL-2) infected vaccine cells showed a significantly enhanced cytotoxic activity against tumor cells in a 4-h ${ }^{51} \mathrm{Cr}$-release assay and in a long-term tumor neutralization assay in vitro.

Having thus provided a 'proof of principle' that a tumor vaccine infected with recombinant NDV producing IL-2 has an added value in comparison to a tumor vaccine infected with rec(-) without IL-2, the question may be asked as to the advantages of NDV as vector for transfer of a therapeutic gene into a tumor. The most important advantages of this vector are its tumor selectivity of viral replication and its safety (12). When comparing viral $\mathrm{M}$ gene expression by RT-PCR we found an about 10,000-fold higher amount in tumor cells as compared to normal cells after infection by NDV (Wilden et al, unpublished data). This would also be the factor of increase of IL-2 production upon infection of human tumor cells with rec(IL-2). Since the virus activates a stronger type I interferon response in normal cells than in tumor cells, its replication in normal cells is inhibited by antiviral genes (10). Potential negative side effects on normal cells are therefore only small. Other reasons for its general safety are its replication in the cytoplasm without a DNA integrating phase so that genotoxic effects can be excluded. Genetic stability is a further advantage of this vector. In this study, the foreign gene product was biologically active and stable.

The future application of rec(IL-2) virus would be either intratumoral application or production of a live cell ATV-NDV tumor vaccine. Both forms of application would aim at producing tumor cells with improved immunogenicity to stimulate adaptive $\mathrm{T}$ cell mediated anti-tumor immunity. This concept is corroborated by the importance IL-2 signals have for priming and secondary expansion of memory $\mathrm{T}$ cells (22-24). The best long-term anti-tumor effects would be expected from $\mathrm{T}$ cells, which have specificity for tumor associated antigens (TAA) such as memory T cells, CD4 helper T cells and CD8 cytotoxic T cells.

We showed in this study that a tumor vaccine infected with rec(IL-2) virus could stimulate $\mathrm{T}$ cells from normal healthy donors as well as $\mathrm{T}$ cells from cancer patients in vitro. In the first case, the vaccine cells were derived from an allogeneic human tumor cell line. Stimulation with a vaccine that produced IL-2 showed significantly increased numbers of CD4 and CD8 T cells expressing the early activation marker CD69 and producing interferon- $\gamma$. In the latter case the tumor cells were derived from autologous tumor cell cultures of HNSCC patients and used to stimulate autologous T cells isolated from patient derived lymph nodes. We only used $\mathrm{T}$ cells and tumor vaccine cells and no dendritic cells in this ELISPOT assay. T cells from all four patients showed a positive ELISPOT response upon stimulation with rec(IL-2) infected autologous tumor vaccine cells. Such a response was not obtained with an unrelated tumor line (U937) infected by the same virus suggesting that autologous tumor antigens had to be present in the assay to generate a memory response. This conclusion was further supported when we increased the stimulatory capacity of the tumor cells by attachment of bsHN-CD28 providing CD28 mediated co-stimulatory signals to $\mathrm{T}$ cells. We saw a further augmentation of the response to the autologous but not to the heterologous vaccine. When testing viral infectivity and attachment of the bispecific molecules it turned out that the heterologous tumor cells were even better than the autologous tumor cells. We conclude that the ELISPOT response was based on TAAspecific memory $\mathrm{T}$ cells and could be augmented when the ATV-NDV vaccine provided further signals via the IL-2 receptor (CD25) and via CD28.

We thus propose the design of an improved second generation ATV-NDV vaccine for use in future studies in cancer patients. This proposition is corroborated by all our previous studies with the ATV-NDV vaccine in which we used the wild-type lentogenic NDV strain Ulster. Recently we obtained results from a randomized prospective trial of adjuvant active specific immunization with ATV-NDV after resection of liver metastases in colorectal cancer patients (25). The study revealed that the ATV-NDV vaccine was effective in colon carcinoma patients in that it significantly improved the 10 year-overall survival as well as the 10-year recurrence-free survival (24). This type of vaccine was also applied to HNSCC cancer patients in whom we achieved a 5year survival rate well above that expected from standard therapy (6). In this study the T cell response of four HNSCC patients to ATV-NDV could be increased by infection of the vaccine cells with the rec(IL-2) virus. We do not expect that the introduction of the foreign gene IL-2 creates unwanted side effects because this would be a highly tumor-targeted approach. There would be only a transient gradient of IL-2 around the vaccination site or within the tumor after intratumoral inoculation.

Recent data demonstrate normal expansion, contraction and memory generation but aberrant secondary responses by CD8 $\mathrm{T}$ cells that lack expression of CD25, the high affinity chain of the IL-2 receptor (23). Therefore, IL-2 seems to be an essential input signal of the CD8 $\mathrm{T}$ cell response programme that leads to effective generation of CD8 T cell responses. In our studies this is corroborated by the increased expression of perforin, cell surface exposed CD107a and cytotoxic potential by $\mathrm{CD} 8 \mathrm{~T}$ cells stimulated with the vaccine infected with IL-2 producing virus. We recently reported on $\mathrm{T}$ cell 
co-stimulation via tumor targeted IL-2 immunocytokines (26). Tumor vaccine cells were modified by infection with NDV and subsequent attachment of defined amounts of virus-specific antibody-IL-2 immunocytokines. Naïve human $\mathrm{T}$ cells could be primed by a tumor vaccine optimally modified by virus infection and attachment of virus specific fusion proteins providing $\mathrm{T}$ cell receptor mediated signal 1 and co-stimulatory signals via the IL-2 receptor (CD25) and via CD28 to perform strong and durable bystander anti-tumor effects in vitro (26). Thus, anti-tumor activity in human $\mathrm{T}$ cells could be induced by stimulation with an NDV infected tumor vaccine producing IL-2 or by a vaccine with cellsurface exposed IL-2 via attachment of virus-specific IL-2 immuno-cytokines. We showed before that NDV infection of tumor stimulator cells leads to a B7-1/B7-2-independent co-stimulation and to the induction of a CD28-responsive element (27). Now we show further augmentation of costimulatory activity if the vaccine provides additional activation signals through CD25. This resulted in improved ELISPOT responses from $T$ cells of cancer patients. We thus propose the design of a second generation vaccine based on ATV-NDV modified by infection with recombinant NDV with an incorporated therapeutic gene such as IL-2 and by the attachment of anti-CD28 bispecific antibodies. The combination of co-stimulatory signals through CD25 and CD28 was shown to induce the most durable $\mathrm{T}$ cell mediated anti-tumor activity (26).

\section{References}

1. Peeters BP, De Leeuw OS, Koch G and Gielkens AL: Rescue of Newcastle disease virus from cloned cDNA: evidence that cleavability of the fusion protein is a major determinant for virulence. J Virol 73: 5001-5009, 1999.

2. Heicappell R, Schirrmacher V, von Hoegen P, Ahlert T and Appelhans B: Prevention of metastatic spread by postoperative immunotherapsy with virally modified autologous tumor cells. I. Parameters for optimal therapeutic effects. Int J Cancer 37: 569-577, 1986.

3. Schirrmacher V: Clinical trials of antitumor vaccination with an autologous tumor cell vaccine modified by virus infection: improvement of patient survival based on improved antitumor immune memory. Cancer Immunol Immunother 54: 587-598, 2005.

4. Ockert D, Schirrmacher V, Beck N, et al: Newcastle disease virus-infected intact autologous tumor cell vaccine for adjuvant active specific immunotherapy of resected colorectal carcinoma. Clin Cancer Res 2: 21-28, 1996.

5. Ahlert T, Sauerbrei W, Bastert G, et al: Tumor-cell number and viability as quality and efficacy parameters of autologous virusmodified cancer vaccines in patients with breast or ovarian cancer. J Clin Oncol 15: 1354-1366, 1997.

6. Karcher J, Dyckhoff G, Beckhove P, et al: Antitumor vaccination in patients with head and neck squamous cell carcinomas with autologous virus-modified tumor cells. Cancer Res 64: 8057-8061, 2004.

7. Steiner HH, Bonsanto MM, Beckhove $\mathrm{P}$, et al: Antitumor vaccination of patients with glioblastoma multiforme: a pilot study to assess feasibility, safety, and clinical benefit. J Clin Oncol 22: 4272-4281, 2004.

8. Becker JC, Pancook JD, Gillies SD, Furukawa K and Reisfeld RA: $\mathrm{T}$ cell-mediated eradication of murine metastatic melanoma induced by targeted interleukin 2 therapy. J Exp Med 183: 2361-2366, 1996

9. Xiang R, Lode HN, Dolman CS, et al: Elimination of established murine colon carcinoma metastases by antibody-interleukin 2 fusion protein therapy. Cancer Res 57: 4948-4955, 1997.
10. Fiola C, Peeters B, Fournier P, Arnold A, Bucur M and Schirrmacher V: Tumor selective replication of Newcastle disease virus: association with defects of tumor cells in antiviral defence. Int J Cancer 119: 328-338, 2006.

11. Schirrmacher V, Ahlert T, Probstle T, et al: Immunization with virus-modified tumor cells. Semin Oncol 25: 677-696, 1998.

12. Schirrmacher V, Haas C, Bonifer R, Ahlert T, Gerhards R and Ertel C: Human tumor cell modification by virus infection: an efficient and safe way to produce cancer vaccine with pleiotropic immune stimulatory properties when using Newcastle disease virus. Gene Ther 6: 63-73, 1999.

13. Zhao H, Janke M, Fournier P and Schirrmacher V: Recombinant Newcastle disease virus expressing human interleukin-2 serves as a potential condidate for tumor therapy. Virus Res, June 4, 2008

14. Janke M, Peeters B, De Leeuw O, Moorman R, Arnold A, Fournier P and Schirrmacher V: Recombinant Newcastle disease virus (NDV) with inserted gene coding for GM-CSF as a new vector for cancer immunogene therapy. Gene Ther 14 : 1639-1649, 2007

15. Haas C, Lulei M, Fournier P, Arnold A and Schirrmacher V: A tumor vaccine containing anti-CD3 and anti-CD28 bispecific antibodies triggers strong and durable antitumor activity in human lymphocytes. Int J Cancer 118: 658-667, 2006.

16. Ertel C, Millar NS, Emmerson PT, Schirrmacher V and von Hoegen P: Viral hemagglutinin augments peptide-specific cytotoxic T cell responses. Eur J Immunol 23: 2592-2596, 1993.

17. Schirrmacher V, Haas C, Bonifer R and Ertel C: Virus potentiation of tumor vaccine $\mathrm{T}$-cell stimulatory capacity requires cell surface binding but not infection. Clin Cancer Res 3: 1135-1148, 1997.

18. Betts MR, Brenchley JM, Price DA, De Rosa SC, Douek DC, Roederer M and Koup RA: Sensitive and viable identification of antigen-specific $\mathrm{CD}^{+} \mathrm{T}$ cells by a flow cytometric assay for degranulation. J Immunol Methods 281: 65-78, 2003.

19. Feuerer M, Beckhove P, Bai L, et al: Therapy of human tumors in NOD/SCID mice with patient-derived reactivated memory $\mathrm{T}$ cells from bone marrow. Nat Med 7: 452-458, 2001.

20. Schmitz-Winnenthal FH, Volk C, Z'Graggen K, et al: High frequencies of functional tumor-reactive $\mathrm{T}$ cells in bone marrow and blood of pancreatic cancer patients. Cancer Res 65: 10079-10087, 2005.

21. Haas C, Lulei M, Fournier P, Arnold A and Schirrmacher V: T-cell triggering by CD3- and CD28-binding molecules linked to a human virus-modified tumor cell vaccine. Vaccine 23: 2439-2453, 2005

22. Jin P, Wang E, Provenzano M, Stroncek D and Marincola FM: Gene expression signatures of interleukin-2 in vivo and in vitro and their relation to anticancer therapy. Crit Rev Immunol 27: 437-448, 2007.

23. Williams MA, Tyznik AJ and Bevan MJ: Interleukin-2 signals during priming are required for secondary expansion of $\mathrm{CD}^{+}$ memory T cells. Nature 441: 890-893, 2006.

24. Yang Y, Xiu F, Cai Z, Wang J, Wang Q, Fu Y and Cao X: Increased induction of antitumor response by exosomes derived from interleukin-2 gene-modified tumor cells. J Cancer Res Clin Oncol 133: 389-399, 2007.

25. Schulze T, Kemmner W, Weitz J, Wernecke KD, Schirrmacher V and Schlag PM: Efficiency of adjuvant active specific immunization with Newcastle disease virus modified tumor cells in colorectal cancer patients following resection of liver metastases - results of a prospective randomized trial. Cancer Immunol Immunother (In press).

26. Aigner M, Janke M, Lulei M, Beckhove P, Fournier P and Schirrmacher V: An effective tumor vaccine optimized for costimulation via bispecific and trispecific fusion proteins. Int $\mathbf{J}$ Oncol 32: 777-789, 2008.

27. Termeer CC, Schirrmacher V, Brocker EB and Becker JC: Newcastle disease virus infection induces B7-1/B7-2-independent T-cell costimulatory activity in human melanoma cells. Cancer Gene Ther 7: 316-323, 2000. 